\title{
PERSONENREGISTER
}

Autorenangaben und Namensnennungen, die nur als Quellenbelege aufgeführt sind, wurden nicht berücksichtigt. Fürsten und übrige Herrscher sind unter ihrem

Vornamen eingeordnet.

Abbadie, Jacques 183, 220f., 228

Achard, Antoine 93

Agoult, François d' 42

Alembert, Jean Baptiste le Rond d' 221

Amalie-Elisabeth, Landgräfin von Hessen-Kassel/Amélie-Élisabeth, landgravine de Hesse-Cassel (16261693) 38,43

Ancillon, Charles 171, 178, 183, 185, 222f.

Ancillon, David 171

Andreae, Johann Valentin 216

Anières, Paul Loriol d' 176

Aristophanes/Aristophane 222

Arius 213

Artis, Gabriel d' 228

Arzberger, Johann Friedrich 115

Assas, François d' 42

Augier, Familie/famille 93

August II., König von Polen/Auguste II, roi de Pologne siehe/voir Friedrich August I./Frédéric-Auguste $\mathrm{I}^{\text {er }}$

Aumeras, Witwe/veuve 200

Aunet 97f

Ausin, Louis d' 234

Baleux, Jean 44

Bannet, Élisabeth 28

Barbeyrac, Jean 213, 222f., 227, 230

Barfuss, de 78

Bamouin, Familie/famille 93

Bastide, Familie/famille 91, 93, 99, 104

Bayle, Pierre 220, 224f., 236

Beaucastel, Familie/famille 94, 105

Beausobre, Charles Louis de 92

Beausobre, Isaac de 219f., 228-230, 235, 237

Beausobre, Louis de 234

Beauvais, Louis Albert $207 f$.
Bellay, Joachim du 233

Berckin, Jean 44

Bérenger, Frédéric de, Herr von Beaufain und Freiherr von Violès/seigneur de Beaufain et baron de Violes $90 \mathrm{f}$., 99

Bergier d'Alençon, Alexandre de $90 \mathrm{f}$.

Bergier d'Alençon, François de 91

Bernard, Fréderic-Henri 92, 105

Bertrand, Jean 44

Bertrand, Marie 96

Betac, Jean 204, 206

Beyer, Hartmann 36

Bismarck, Otto von 184

Blancart/Blancquard, Pierre 29

Blancart/Blancquard, Abraham 29

Bodin, Jean 134

Borcke, Heinrich Adrian Graf von/comte de 175

Bossuet, Jacques Bénigne 215, 221

Boué, Jacques 132

Boué, Pierre 127, 129-132, 134

Boué, Susanne 129

Bouhours, Dominique 233

Bouillon, Herzog von/duc de 18

Bourguet, Louis und Familie/et sa famille 93, 105

Bourguet, Sekretär/secrétaire 91

Boyer, Händler in Hamburg/commerçant à Hambourg 134

Boyer, Refugié in Berlin/réfugié à Berlin 87

Brandt, Eusebius von, Staatsrat/conseiller d'État 78, 86

Brehé, Refugié in Berlin/réfugié à Berlin 87

Brésillon, Pierre 96

Breton, Familie/famille 87

Briasson, Buchhändler/libraire 237

Brunel, Antoine 44

Brunel, Étienne 44

Burlamaqui, Jean-Jacques 223 
Calvin, Jean 7, 141, 194

Campagne, Alexandre Auguste de 177, 179

Cargès, Otto 86

Castagnier, Pierre 40

Cayard, Louis 211

Cazalét, Jean $205 \mathrm{f}$.

Chalanqui, Schlosser/serrurier 93

Chalon, Guillaume de 91

Chambaud, Louis 90

Chapat, Louis 100

Charlotte, Landgräfin von Hessen-

Kassel/landgravine de Hesse-Cassel (1627-1686) 21

Chatain, Suzanne $96 \mathrm{f}$.

Chauvet, Noé 90

Chauvin, Étienne 223-226, 230

Chazot 236

Chièze, Paul 90, 104

Chièze, Phillippe 100

Chion, Gaspard 91, 98, 101, 105

Chodowiecki, Daniel 211

Christian Ernst, Markgraf von

Brandenburg-Bayreuth/margrave de

Brandebourg-Bayreuth (1644-1712)

107-114, 117-119, 121f.

Christian I., Kurfürst von

Sachsen/Christian I ${ }^{\mathrm{IT}}$, électeur de Saxe (1560-1591) 52

Clignet, Henri 28f., 32

Clignet, Jacques 28

Clignet, Nicolas 28

Clignet, Pierre 28

Cnipius Andronicus, Jean 36

Cochius, Herman 86

Colbert, Jean-Baptiste 185

Coninxloo, Adrian von 28

Convenent, Gabriel de 91, 103

Convenent, Jean 91, 98, 101f., 104

Cooper, Anthony Ashley, III. earl of

Shaftesbury (1671-1713) 221

Cordier, Philippe 204, 206

Cornand 90

Corneille, Pierre 141

Costa, Duarte Nunes da 133

Coste, Pierre 219, 221, 227

Coudray, François 59

Courselle, Jacqueline de 41

Couston, Judith 96

Crès, Magdelaine 100

Cumberland, Richard 223
Danckelmann, Eberhard Christoph

Balthasar von $78,86,216,224 f$.

Dantal, Charles 236

Daubigny, Pierre 73

Deckenberger, Hans 29

Degenfeld, Luise von 21

Descartes, René 21

Dewert, Marin 90-92, 104

Diderot, Denis 221, 229, 239

Dohna, Alexander von 78f., 222

Dörnberg, Wolfgang Ferdinand Freiherr von/baron de 178

Drouet, Jean 86

Drouin 26

Du Bos, Abt/l'abbé 233

Du Cros, Joseph August 110-114, 119 , 122

Dubois, Gaspard 91, 103

Ducros, Familie/famille 93

Dufour, Jacques Ferdinand, Freiherr/baron Dufour von/de Feronce 66

Dufour, Jacques Marc Antoine oder/ou Marc Antoine 65f

Durnas, Jean Louis Alexandre 67

Dumont, Gabriel 59

Duras, Herzog von/duc de 41

Duvigneau, David Antoine 65,67

Duvigneau, Gabriel Philippe 66

Ebrard, August 108

Elisabeth Stuart, Tochter König Jakobs I. von England/Élisabeth Stuart, fille du roi Jacques I" d'Angleterre (15961662) 18

Elisabeth-Charlotte, Tochter Karl Ludwigs, Kurfürst von der Pfalz/Élisabeth-Charlotte, fille de l'électeur palatin Charles-Louis 21

Erichs, Peter 129

Erman, Jean-Pierre 171-192, 226

Ewald Friedrich, Graf von Hertzberg/comte de Hertzberg (17251795) 179

Fabre, Witwe/veuve 90

Faravel, Balthazard 88

Farre, Abt/l'abbé de la 236

Feautrier, Jeanne 90

Félix, Pierre 93, 104

Ferdinand II., Kaiser/Ferdinand II, empereur (1578-1637) 8, 53 


\section{Ferrand 93}

Feuquier, Jean 73

Feuquière, Jean Baptiste 73

Fiers, Jeanne 96

Firmin 90

Fontenelle, Bernard Le Bouyer de 233, 236

Fontius, Martin 229

Formey, Jean Henri Samuel 176, 221 , $223,228-230,234,236-238$

Fourmont, Étienne de 233

Fournier, Arnail 131

Fournier, Simon 131

Francezon, Pierre 93, 101

Francke, Hermann August 214

Frege, Christian Gottlob 66

Friedrich August I., der Starke, Kurfürst von Sachsen/Frédéric-Auguste $I^{\mathrm{er}}$, le Fort, électeur de Saxe (1670-1733) 5162

Friedrich August II., Kurfürst von Sachsen/Frédéric-Auguste II, électeur de Saxe (1696-1763) 60

Friedrich August III. Kurfürst von Sachsen/Frédéric-Auguste III, électeur de Saxe (1750-1827), seit 1806 als Friedrich August I. König von Sachsen 68

Friedrich I., König in Preußen/Frédéric $\mathrm{I}^{\mathrm{er}}$, roi de Prusse (1657-1713) 57, 77, 79, $81,86,97,99,144,172,180,196$, 214

Friedrich I., Landgraf von Hessen-Kassel, König von Schweden/Frédéric I I landgrave de Hesse-Cassel, roi de Suedde (1676-1751) 74f.

Friedrich II., der Große, König von Preußen/Frédéric II, le Grand, roi de Prusse (1712-1786) 68, 146f., 164, 186, 211, 235f., 239

Friedrich II., Landgraf von HessenHomburg/Frédéric II, landgrave de Hesse-Hombourg (1633-1708) 44

Friedrich II., Landgraf von HessenKassel/Frédéric II, landgrave de HesseCassel (1720-1785) 73

Friedrich III., Kurfürst von Brandenburg, Herzog von Preußen/Frédéric III, électeur de Brandebourg, duc de Prusse, siehe/voir Friedrich I., König in Preußen/Frédéric I ${ }^{\mathrm{tr}}$, roi de Prusse
Friedrich III., Kurfürst von der Pfalz/Frédéric III, électeur palatin (1515-1576) 17, 27

Friedrich IV., Kurfürst von der Pfalz/Frédéric IV, électeur palatin (1574-1610) 18

Friedrich V., Kurfürst von der Pfalz/Frédéric V, électeur palatin (1596-1632) 18

Friedrich Wilhelm I., Kurfürst von Brandenburg, Herzog von Preußen, der Große Kurfürst/Frédéric-Guillaume $I^{\text {er }}$, électeur de Brandebourg, duc de Prusse, le Grand-Électeur (1620-1688) 43, 76, $78,82,142,195,235$

Friedrich Wilhelm I., König in Preußen/Frédéric-Guillaume I ${ }^{\text {er }}$ roi de Prusse (1688-1740) 78, 81-83, $155 f$.

Friedrich Wilhelm II., König von Preußen/Frédéric-Guillaume II, roi de Prusse (1744-1797) 163

Friedrich Wilhelm III., König von Preußen/Fréderic-Guillaume III, roi de Prusse (1770-1840) 149

Friedrich-Heinrich von OranienNassau/Frédéric-Henri d'OrangeNassau (1584-1647) 19, 85

Fuchs, Paul von 218, 222, 225

Gardiol, Suzanne 93

Gaudin, Pierre 90

Gaultier de Saint Blancard, François 212

Geltner, Pierre 36

Gely, Catherine 96

George, Jean 205f.

Gerhard, Paul 214

Glaubourg, Jean 36

Gleize 93

Gourand, Daniel 40

Gourdeau, Claude 90

Goy, Marie 44

Graevius, Johann Georg 216

Grandidier, Pastor/pasteur 33

Granet, André 99

Grotius, Hugo 21, 216, 223f., 228

Grumbkow, Joachim Ernst von 78

Guaimard, Françoise 96

Guichard, Charles Théophile 238

Guillaume 90

Guy, Pierre 86, 105

Guyon, Jeanne 96 
Halcke, Gustav Georg von 73

Harnacks, Adolf von 221

Hazard, Paul 215

Heinrich IV., König von Frankreich/Henri IV, roi de France (1553-1610) 18

Henri-Charles, Herzog von Trémoille, Freiherr von Vitré/duc de la Trémoille, baron de Vitré (1620-1672) 38

Herff, Henri 28

Herff, Marie Élisabeth 28

His, Händler/commerçant 134

Hobbes, Thomas 223

Jacquelot, Isaac 221

Jakob, Andreas 109

Jakob I., König von England/Jacques I"r, roi d'Angleterre (1566-1625) 18

Jammis, Marguerite 96

Jandan, Jacques Égide Duhan de 235

Jariges, Philippe de 235

Jaucourt, Louis de 223

Jean, Denis 41

Johann Georg III., Kurfürst von

Sachsen/Jean-George III, électeur de

Saxe (1647-1691) 52

Johann Georg IV., Kurfürst von

Sachsen/Jean-George IV, électeur de

Saxe (1668-1694) 52

Johann-Kasimir, Pfalzgraf/Jean-Casimir, comte palatin (1543-1592) 18

Jordan, Charles Étienne 232-236, 237

Jordan, Daniel 100

Jordan, Gustav von 193

Juny, Jacques 40

Jurieu, Pierre 212, 223, 228

Kant, Immanuel 229

Karl, Landgraf von Hessen-

Kassel/Charles, landgrave de Hesse-

Cassel (1654-1730) 72-74, 82

Karl Ludwig I., Kurfürst von der Pfalz/Charles-Louis $\mathrm{I}^{\mathrm{rr}}$, électeur palatin (1618-1680) 18, 19-23, 26, 28-30, 33

Karl II. Kurfürst von der Pfalz/Charles II, électeur palatin (1651-1685) 17, 18, 26

Katharina Belgica, Gräfin von Nassau/Catherine Belgia, comtesse de Nassau (1578-1648) 37

Kepler, Johannes 21

Klitzing, Mädchenname/nom de jeune fille: Mangelin 180
La Condamine, Charles Marie de 237

La Houssaye, François de Paule, Ritter, Herr von Sardan, Vicomte von/ chevalier, seigneur de Sardan, vicomte de 72

La Mettrie, Julien Offray de 219, 238, 240

Laigneau, Anne 96

Lalouette, Frédéric de, Ritter von Vernicourt/chevalier de Vernicourt 73

Langes, François de, Freiherr von Lubières/baron de Lubières 99, 103

Lautier, Juwelier/joaillier 232

Laveaux, Jean-Charles Thibault de $165 f$.

Le Clerc, Jean 228

Le Coq, Jacques 57-62

Le Loup, Daniel 26

Le Roy, Pierre 26

Leibniz, Gottfried Wilhelm 215-221, 224f., 230

Lenfant, Jacques 228, 235, 237

Leplat, Raymond 59

Lessing, Johann Gotthold 65

Lipsius, Justus 216

Lith, Gabriel von der 60

Locke, John 221, 223, 227

Loge, Josué de 31

Longuelune, Zacharias 59

Loreilhe, Händler/marchand 127, 134

Louis, Jean, Vater/père 27

Louis, Jean, Sohn/fils 27

Lubière, Freiherr von/baron de $86,90 f$., 97, 99

Ludwig IV. der Bayer, Kaiser/Louis IV de Bavière, empereur (1281/82-1347) 35

Ludwig VI., Kurfuirst von der Pfalz/Louis VI, électeur palatin (1539-1583) 18, 28

Ludwig XIV., König von Frankreich/Louis XIV, roi de France (1638-1715) 7, 21, 26, 39, 54, 80, 85, $125,127,132,140,142,185,187,189$, $195,212,215$

Ludwig XVI., König von Frankreich/Louis XVI, roi de France (1754-1793) 146-148, 153

Ludwig-Philipp von Simmern/LouisPhilippe de Simmern (1602-1655) 17

Luise Henriette von OranienNassau/Louise-Henriette d'OrangeNassau (1627-1667) 85

Lukrez/Lucrèce 222

Luther, Martin 18, 189 
Malbran, Jean 205, 206

Mallein, Suzon 44

Marconnay, Familie/famille 180

Maréchal, Bernard 90

Marot, Clément 233

Marperger, Jacob 35, 53

Mary Tudor, Königin von England/Marie

Tudor, reine d'Angleterre (1496-1533) 36

Maupertuis, Pierre Louis Moreau de 219

Maurau, Moyse 90

Mayet 181

Melanchton, Philipp/Mélanchton, Philippe 36

Mendelssohn, Moses 229

Mercier, Jean Pierre 58

Merian, Christoph 43, 48

Merian de la Grivelière, Charles Gustave 86

Michel, Marie 93

Mirabeau, Honoré Gabriel Riqueti, Graf von/comte de 181

Mirmand, Henri, Marquis von/marquis de (1650-1721) 72, 74, 79

Mollerus, Pierre 28

Monlaret, Jean 42

Montfaucon, Bernard de, Benediktiner/bénédictin 233

Moritz, Kurfürst von Sachsen/électeur de Saxe (1521-1553) 54

Moritz, Landgraf von HessenKassel/landgrave de Hesse-Cassel (1572-1632) 72

Moritz, Pfalzgraf/comte palatin (16211652) 19

Mösch, Andreas 118, 121

Muralt, Béat Louis de 232

Napoleon I., Kaiser der

Franzosen/Napoléon $\mathrm{I}^{\mathrm{r}}$, empereur des

Français (1769-1821) 176

Naudé, Philippe, Vater/père 225

Naudé, Philippe, Sohn/fils 220

Nemeitz, Joachim Christoph 232

Neufville, Pierre de 29

Newton, Isaac 221

Nicéron, Jean-Pierre 233

Nicola, Daniel 202

Noé, Jean Jacob 205f.

Noodt, Gerhardt 223

Nouvel, Jean 44

Nouvel, Pierre 44
Olevianus, Gaspard 17

Ottheinrich, Kurfuirst von der

Pfalz/Othon-Henri, électeur palatin (1502-1559) 17

Ougier, Jean 92, 104, 105

Ougier, Jeanne 92

Pajon, Louis Élie 234

Pelagius/Pélage 213

Pelet, André 92, 104

Pellet, Jean $129 \mathrm{f}$.

Pepliers, Robert Jean des 200

Pérard, Jacques de 234

Péricard, Sébastien Salomon 30

Petit, Charles 86, 90f., 98, 101, 104

Pez, Ozias 98

Philipp I., Landgraf von Hessen-

Kassel/Philippe $I^{\mathrm{er}}$, landgrave de HesseCassel (1504-1567) 71f.

Philipp II., König von Spanien/Philippe II, roi d'Espagne (1527-1598) 17

Philipp von Orleans/Philippe d'Orléans (1674-1723) 21

Philipp Ludwig II., Graf von HanauMünzenberg/Philippe-Louis II, comte de Hanau-Münzenberg (1576-1612) 37

Philipp Wilhelm Prinz von Oranien/Philippe-Guillaume prince d'Orange (1554-1618) 91

Poiret, Pierre 33

Poitevin, Samuel 33

Polonus, Martinus 225

Ponnier, Jean Pierre 114, 122

Pouillon, Anne 27

Poullain, Valérand 36

Pourvoyeur, Jean 27

Prémontval, André-Pierre Le Guay de $165 f$.

Pufendorf, Samuel von 216, 219, 222227

Quentin, Adam 93

Rabaut Saint-Étienne, Jean-Paul 173f

Raby, Thomas Wentworth, baron, earl of Strafford $89 \mathrm{f}$

Racine, Jean 141

Raynal, Abt/abbé 174-175, 181

Réaumur, René-Antoine Ferchault de 233 
Rebeur, Korrespondent in Schwedt, Uckermark/correspondant à Schwedt, Marche Ukraine 180

Reclam, Pierre Chrétien Frédéric 163, 171-192, 226

Richelieu, Armand Jean du Plessis, Herzog von/duc de 7, 19

Richter, Johann Adolph $65 f$.

Rion, Dominique 129

Ritter, Mathias 36

Robert, Joseph 96

Rochau, von 73

Rollin, Charles 233, 236

Rosales, Jacob 133

Rost, Pastor/pasteur 28

Rotenschild 21

Rousseau, hugenottischer Immigrant/immigrant huguenot 87

Rousseau, Jean-Jacques 183, 229, 239

Roux, Jacob 164

Roux, Jeanne 96

Ruat, Guillaume 91, 104

Rupert, Pfalzgraf bei Rhein/comte palatin du Rhin (1619-1682) 19

Saint-Ange, Anne 99

Saint-Blancard, Gaultier de 183

Saint-Laurent/Saint-Laurens, Louis de 91

Sanchon, oranischer Refugié in

Berlin/réfugié orangeois à Berlin 93

Sardan, Ritter Herr von/chevalier seigneur de 72

Sarre, Pierre 205f.

Sauzin, Françoise de 99

Sauzin, Joseph de 103

Schanz, Georg 108

Schlüter, Rat/conseiller 177

Schomberg, Friedrich Hermann Graf von/comte de 183

Sémeri, Suzanne 44

Serres, Jean-Jacques 90, 104, 105

Sévigné, Marie de Rabutin-Chantal, Marquise von/marquise de 220

Silvestre, Louis de 59

Simons, Richard 225, 228

Skytte, Benedikt von 216

Sophie Charlotte, Königin in Preußen/reine de Prusse (1668-1705) 91

Spanheim, Ezechiel von 78, 225

Spener, Philipp Jakob 214, 216

Spinoza, Baruch 21
Stosch, Marie-Charlotte 176

Sy, Pierre 205

Teissier, Antoine 224

Teissier, Ève 93

Thiébault, Dieudonné 175

Thieriot, Jacques Henri 66

Thiriot, Nicolas-Claude 236

Thomasius, Christian 214, 216, 223

Tourtin, Pastor/pasteur 41

Trublet, Nicholas-Charles-Joseph,

Abt/l'abbé 237

Ursinus, Zacharias/Zacharie 17

Vasseur, Antoine 40

Vattel, Emer de 238

Vernet, Jacob 238

Veyne, Paul 179

Veyssière La Croze, Mathurin 232, 235

Vigut, Annibal 101, 105

Villegagnon, Ritter/chevalier 41

Vioujas 89

Viset, französischer Diplomat in

Brandenburg-Bayreuth/diplomate

français à Brandebourg-Bayreuth 114

Voltaire, François-Marie Arouet de 165 , $187,219,229,236,239$

Voulaire, Händler/marchand $100 \mathrm{f}$.

Wallin, Georg 232

Warin, Anthoine 29

Wilhelm, Nicolas 28

Wilhelm I. von Oranien-

Nassau/Guillaume I ${ }^{\text {er }}$ d'Orange-Nassau (1533-1584) 37

Wilhelm III. von Oranien-Nassau, König von England/Guillaume III d'OrangeNassau, roi d'Angleterre (1650-1702) 43, 79, $85 f$., 97

Wilhelm V., Landgraf von HessenKassel/Guillaume V, landgrave de Hesse-Cassel (1602-1637) 38

Wolff, Schulmeister/maître d'école 30

Wolff, Christian 188, 219, 221, 223, 229, $236 \mathrm{f}$.

Wolzogen, Christoph Andreas von 29

Zeller, Moritz 30

Zinzerling, Juste 232

Zollikofer, Georg Joachim 64, 67 